\title{
IGF2BP3 (IMP3) expression is a marker of unfavorable prognosis in ovarian carcinoma of clear cell subtype
}

\author{
Martin Köbel ${ }^{1,2}$, Haodong $\mathrm{Xu}^{3}$, Patricia A Bourne ${ }^{3}$, Betsy O Spaulding ${ }^{4}$, Ie-Ming Shih ${ }^{5}$, \\ Tsui-Lien Mao ${ }^{6}$, Robert A Soslow ${ }^{7}$, Carol A Ewanowich ${ }^{8}$, Steve E Kalloger ${ }^{1}$, Erika Mehl ${ }^{1}$, \\ Cheng-Han Lee ${ }^{1}$, David Huntsman ${ }^{1}$ and C Blake Gilks ${ }^{1}$ \\ ${ }^{1}$ Department of Pathology, Genetic Pathology Evaluation Centre of the Prostate Research Centre, Vancouver \\ General Hospital and British Columbia Cancer Agency, Vancouver, BC, Canada; ${ }^{2}$ Institute of Pathology, \\ Charite Hospital, Berlin, Germany; ${ }^{3}$ Department of Pathology and Laboratory Medicine, University of \\ Rochester Medical Center, Rochester, NY, USA; ${ }^{4}$ Dako North America, Carpinteria, CA, USA; ${ }^{5}$ Department of \\ Pathology, Johns Hopkins University, Baltimore, MD, USA; ${ }^{6}$ Department of Pathology, National Taiwan \\ University Hospital, Tapei, Taiwan; ${ }^{7}$ Surgical Pathology, Memorial Sloan-Kettering Cancer Center, New York, \\ NY, USA and ${ }^{8}$ Department of Laboratory Medicine and Pathology, University of Alberta and Royal Alexandra \\ Hospital, Edmonton, AB, Canada
}

\begin{abstract}
Clear cell carcinoma is an uncommon subtype of ovarian carcinoma, accounting for $10 \%$ of cases. Clear cell carcinoma typically presents with stage I or II disease, and in this setting prognostic markers could aid in management decisions, in particular the decision to treat with adjuvant chemotherapy. We tested whether expression of insulin-like growth factor 2 mRNA-binding protein 3 (IGF2BP3, also known as IMP3) can serve as a new biomarker to predict outcome for patients with clear cell carcinoma and other subtypes of ovarian carcinoma. The expression of IGF2BP3 was evaluated by immunohistochemistry in $\mathbf{4 7 5}$ ovarian carcinomas of different subtypes and correlated with disease-specific survival. IGF2BP3 antibody specificity was validated by correlation of IGF2BP3 protein with mRNA expression level in a series of 35 ovarian carcinomas $(r=0.849$, $\boldsymbol{P}<\mathbf{0 . 0 0 0 1 )}$. IGF2BP3 protein expression was an independent marker of reduced disease-specific survival (risk ratio $2.9,95 \%$ confidence interval $1.4-5.8)$ in the clear cell subtype $(N=128)$, but not in high-grade serous $(N=198)$ or endometrioid $(N=121)$ carcinomas. The prognostic significance of IGF2BP3 expression for reduced disease-specific survival (risk ratio 2.6, 95\% confidence interval 1.3-5.0) was confirmed in an independent series of cases $(N=150)$ from three different centers in North America. We conclude that IGF2BP3 is the first biomarker of prognostic significance in ovarian clear cell carcinoma that has been validated in an independent case series.

Modern Pathology (2009) 22, 469-475; doi:10.1038/modpathol.2008.206; published online 9 January 2009
\end{abstract}

Keywords: ovarian clear cell carcinoma; IGF2BP3; IMP3; prognosis; immunohistochemistry; cancer

The insulin-like growth factor mRNA-binding protein family comprises three proteins (IGF2BP1-3), which regulate mRNA transport, translation, and turnover by binding the coding regions of target mRNAs such as IGF2 (insulin-like growth factor 2), MYC, and ACTB ( $\beta$-actin). ${ }^{1-4}$ IGF2BP expression is almost exclusively restricted to embryogenesis, with

Correspondence: Professor CB Gilks, MD, Department of Pathology, Vancouver General Hospital, Room 1207 1st floor JPPN, 855 West 12th Ave, Vancouver, BC, Canada V5Z 1M9.

E-mail: Blake.Gilks@vch.ca

Received 18 July 2008; revised 3 November 2008; accepted 4 November 2008; published online 9 January 2009 little or no detectable protein in normal adult tissues. ${ }^{5}$ We have recently shown that IGF2BP1 (alias IMP1) is overexpressed in high-grade ovarian carcinomas of serous and clear cell subtypes. ${ }^{6}$ Another family member, IGF2BP3, was cloned from a pancreatic tumor cDNA screen and was originally designated as KOC (KH-domain-containing protein overexpressed in cancer). ${ }^{7}$ IGF2BP3 expression has been associated with an unfavorable outcome in renal clear cell carcinoma. ${ }^{8,9}$ On the basis of this finding, we postulated that IGF2BP3 expression could also be a prognostic indicator for ovarian clear cell carcinoma, a tumor type with morphologic similarities to renal clear cell carcinoma. 
Together with endometrioid carcinomas, clear cell carcinomas are the second most common subtype of ovarian carcinomas after high-grade serous subtype. They account for $10 \%$ of all cases, and are mostly $(80 \%)$ diagnosed at stage I or II. $^{10,11}$ Women diagnosed with ovarian clear cell carcinomas have a 5-year overall survival ranging from $88 \%$ in stage I to $33 \%$ in stage III. $^{12}$ Except for stage, there are no validated prognostic factors for clear cell carcinoma and their behavior is unpredictable and in some cases follow an aggressive clinical course. Histopathological grading, for example, is not used as it has consistently been shown to be of no prognostic significance; ${ }^{11}$ as such all clear cell carcinomas are considered to be grade III. ${ }^{13}$ In the absence of validated prognostic factors for clear cell carcinoma, we sought to determine whether expression of IGF2BP3 correlates with prognosis for this distinct subtype.

\section{Materials and methods}

\section{Patients and Tumor Specimens}

IGF2BP3 expression was assessed for 475 ovarian carcinomas from a population-based cohort from British Columbia and a validation set of 150 ovarian clear cell carcinomas from three other institutions of North America: these cohorts were described earlier. ${ }^{14}$ Briefly, the cohort from British Columbia was obtained from a population of approximately four million people in British Columbia. For the period 1984-2000, 2555 patients with ovarian carcinoma were registered in the Cheryl Brown Ovarian Cancer Outcomes Unit, which records a large majority of cases of ovarian carcinomas in the region. A total of 834 patients without macroscopic residual disease after surgery were selected for further study. Ninetyone patients with excellent prognosis (grade 1, stage $1 \mathrm{a}$ or $1 \mathrm{~b}$ ) were excluded from the study; only $3 \%$ of women in this group died of disease during the follow-up period. As clear cell carcinomas are by definition grade III, no clear cell carcinomas were excluded. After a full gynecopathological review ${ }^{11}$ according to WHO criteria, ${ }^{13} 541$ tissue blocks were available and used for tissue microarray construction. A representative area of each tumor was selected and duplicate $0.6-\mathrm{mm}$ tissue cores were punched to construct a tissue microarray (Beecher Instruments, Silver Springs, MD, USA). A review after tissue microarray construction revealed that an additional 23 cases were not sampled adequately. Also excluded were 18 cases of rare histological type (including seven undifferentiated, six transitional, and one squamous carcinoma) and five histologically unclassified cases. The serous subtype was further subdivided into low and high grade, ${ }^{15}$ and all 11 low-grade serous carcinomas were excluded because of insufficient numbers for subtype analysis. This selection resulted in a study population of 489 cases belonging to one of the four major cell types (high-grade serous, clear cell, endometrioid, and mucinous). Table 1 shows the clinicopathological data from 475 patients for whom IGF2BP3 expression data were assessable.

The validation set was composed of 150 cases in which pure clear cell carcinomas were selected after a file search in the period from 1980 to 2006. These cases were obtained from three institutions (Johns Hopkins University, Baltimore, MD, USA, $N=69$, University of Alberta, Edmonton, Canada, $N=42$, Memorial Sloan-Kettering Cancer Center, New York, NY, USA, $N=39$ ).

To validate immunohistochemistry by analysis of mRNA expression, fresh frozen as well as paraffinembedded tissue was obtained for 35 ovarian tumors from the Vancouver General Hospital tumor bank from patients who were undergoing surgery during 2004-2005. ${ }^{16}$ These cases include the following subtypes: high-grade serous $(N=27)$, clear cell $(N=3)$, endometrioid $(N=4)$, and one serous borderline tumor. Approval for the study was obtained from the Research Ethics Board (H04-60102).

\section{Treatment and Outcome}

Minimal staging with inspection and palpation of all peritoneal surfaces and the retroperitoneal area, biopsies of any suspect lesions for metastases, peritoneal washing, and infracolic omentectomy was the standard of care in all the four institutions. The majority of patients were treated during a time period when current comprehensive surgical staging was not the standard of care, and information regarding extent of surgical staging is missing in a

Table 1 Clinicopathological characteristics and IGF2BP3 expression of patients with ovarian carcinomas

\begin{tabular}{lcccc}
\hline Cohort by subtype & $\mathrm{N}$ & Age (mean \pm s.d.) & Stage III/IV (\%) & IBP2BP3 expression (\%) \\
\hline British Columbia cohort & 475 & $57.9 \pm 12.8$ & 16.8 & 46.8 \\
$\quad$ High-grade serous & 196 & $60.8 \pm 11.5$ & 33.2 & 5.0 \\
Clear cell & 128 & $56.4 \pm 13.3$ & 4.1 & 51.6 \\
Endometrioid & 121 & $55.5 \pm 13.2$ & 3.3 & 27.3 \\
$\quad$ Mucinous & 30 & $55.6 \pm 13.7$ & & 86.7 \\
Validation set & & & 23.7 & 54.0 \\
$\quad$ Clear cell & 150 & $51.5 \pm 10.8$ & & \\
\hline
\end{tabular}

s.d., standard deviation. 
significant number of cases. Hence, these cohorts must be considered as incompletely staged, with an approximately $25 \%$ risk that residual tumor was left behind after primary surgery. ${ }^{17}$ Patients with ovarian carcinoma from the British Columbia cohort received adjuvant therapy according to the provincial treatment guidelines of the British Columbia Cancer Agency that changed over the years ${ }^{18}$ and are available at http://www.bccancer.bc.ca/PPI/Typesof Cancer/Ovary/default.htm. Similarly, the treatment regimens for ovarian clear cell carcinomas of the other centers were revised over time and are shown in Supplementary Table 1; adjuvant therapy for ovarian clear cell carcinoma was consequently quite heterogeneous. The study endpoint was defined as disease-specific survival. Disease-specific survival was defined as ovarian carcinoma-specific death, in which ovarian cancer was the primary or underlying cause of death. Death from concurrent disease (ie second malignancy) was coded as 'died of other cause'; death resulting from toxicities relating to treatments for ovarian carcinoma was coded as 'died of toxicities' and these patients were censored for disease-specific survival. This information was available for all $(100 \%)$ patients of the British Columbia cohort (Cheryl Brown Ovarian Cancer Outcomes Unit) and $91.3 \%$ of patients from the validation set. Mean follow-up time was 5.9 years (British Columbia cohort) and 4.6 years (validation set).

\section{Immunohistochemistry and Scoring}

Serial $4-\mu \mathrm{m}$ sections were cut for immunohistochemical analysis. After heat-antigen-induced retrieval, staining was carried out on an automated system as per the manufacturer's protocol (DAKO, Carpenteria, CA, USA). The expression of IGF2BP3 in ovarian carcinomas was analyzed by immunohistochemistry using a mouse monoclonal antibody against IGF2BP3 (clone 69.1, dilution 1:100, DAKO, Carpenteria, CA, USA). ${ }^{19}$ Normal fallopian tube, ovarian surface epithelium, ovarian stroma, and endometrium were non-immunoreactive and were used as negative controls. A malignant melanoma sample with known IGF2BP3 expression served as a positive control. IGF2BP3 staining on tissue microarrays was scored by a pathologist (MK) blinded to clinical outcome. The cut-off point for positive cases was any convincing cytoplasmic expression in more than $5 \%$ of tumor cells. Comparison between the results of tissue microarray assessment of IGF2BP3 staining and full-section immunohistochemical assessment was done in 22 cases (20 that were negative on tissue microarray assessment and two that were positive). Results of assessment of full sections were concordant in 21/22 cases; a single case that was negative on tissue microarray assessment showed focal positivity on full section staining.

\section{Antibody Confirmation}

The Human Exonic Evidence Based Oligonucleotide microarray (HEEBO) (Stanford, CA, USA) was used to examine the mRNA expression profiles of 35 ovarian carcinomas. After confirmation of the presence of viable tumor by frozen section, total RNA from the tumor samples was extracted using mirVa$\mathrm{na}^{\mathrm{TM}}$ RNA isolation kit (Ambion, Austin, TX, USA). The total RNA was reverse transcribed into cDNA using a mixture of oligo dT (Operon, HPLC purified) and random hexamer (Amersham, Cat 27-2166-01) primers with incorporation of amino allyl-dUTP (Ambion 8439). Cy3 and Cy5 dyes (Amersham RPN 5661) were used for indirect labeling of the cDNA from reference RNA (Stratagen, Universal Human Reference RNA, Cat 740000) and cDNA from tumor specimens, respectively. Microarray hybridization and washing were carried out using standard procedures. $^{20,21}$ Microarrays were scanned on a GenePix 4000 microarray scanner and fluorescence ratios (tumor/reference) were calculated using GenePix software. Only spots with a ratio of signal over background of at least 1.5 in the Cy5 and 1.5 in the Cy3 channel were included. Gene centering was applied to the expression values for this series of tumors. Our current analysis was restricted to the expression level of IGF2BP3 (IMP3) mRNA and was correlated to the status of protein expression by immunohistochemistry.

\section{Statistical Analysis}

Univariate disease-specific survival analysis was carried out by generating Kaplan-Meier curves and differences were assessed with the log-rank statistic. Multivariable disease-specific survival analysis was assessed with the Cox proportional hazards regression model. Differential expression of IGF2BP3 across the four histopathological subtypes was assessed with contingency analysis and statistical differences were quantified using the Pearson's chisquare statistic. For all analyses, a $P$-value $<0.05$ was considered statistically significant. Statistical analyses were carried out using SPSS software (version 15.0; SPSS, Chicago, IL, USA).

\section{Results}

IGF2BP3 expression by immunohistochemistry correlated strongly with mRNA levels in a set of 35 ovarian carcinomas (Figure $1, r=0.849, P<0.001$ ). IGF2BP3 staining was not observed in sections of normal tissue, including ovarian stroma and surface epithelium, fallopian tube, and endometrium (data not shown).

Forty-seven percent of ovarian carcinomas of the British Columbia cohort showed IGF2BP3 expression (Figure 2, all slides are available online at http://bliss.gpec.ubc.ca/ (under OOU)). IGF2BP3 
472

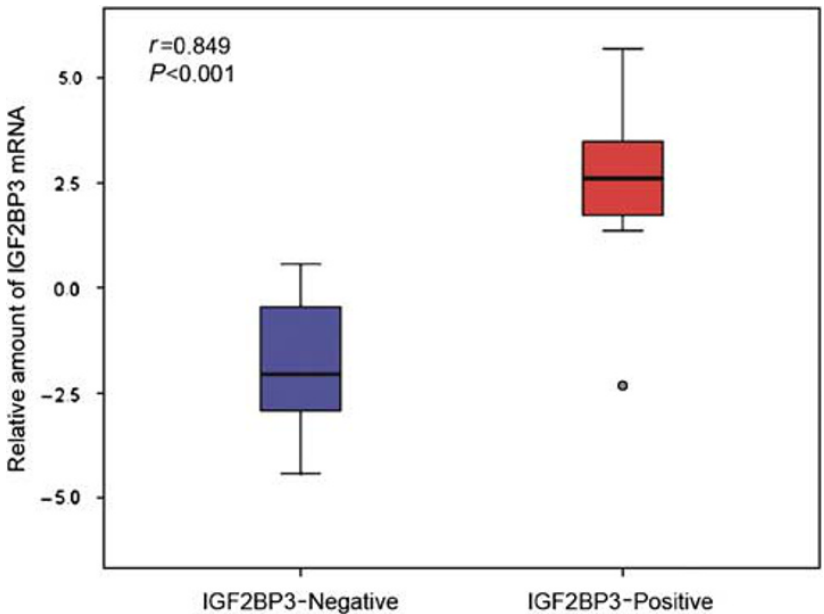

Figure 1 Relative amount of IGF2BP3 mRNA for immunohistochemically IGF2BP3-negative and -positive ovarian carcinomas.

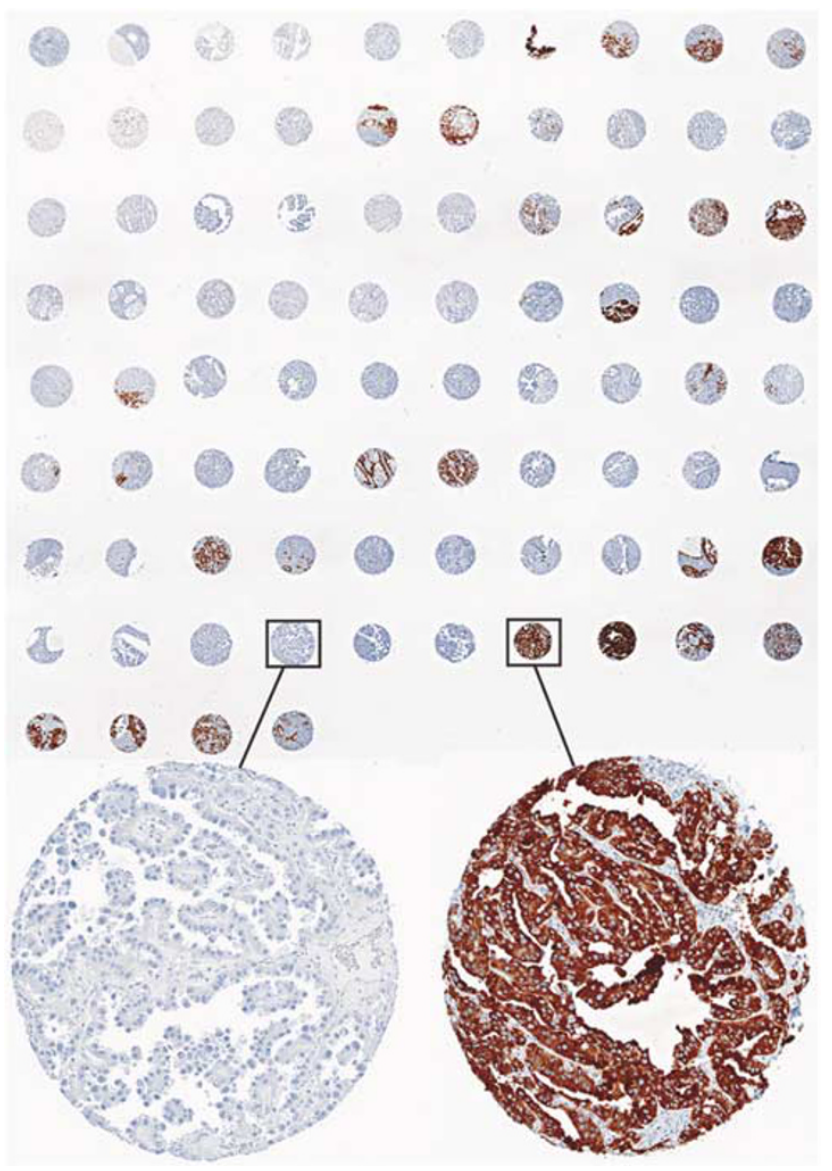

Figure 2 Immunohistochemical stains for IGF2BP3 showing a tissue microarray consisting of ovarian clear cell carcinomas of the validation set. Lower left-a clear cell carcinoma negative for IGF2BP3, lower right-a clear cell carcinoma positive for IGF2BP3.

expression differed between subtypes $(P<0.001$, Pearson's chi-square, Table 1). The highest rate of expression was seen in the mucinous subtypes $(86 \%, N=30)$, followed by clear cell carcinomas
(52\%, N=128) and high-grade serous carcinomas (50\%, $N=198)$, with the lowest expression rate in endometrioid subtype (27\%, N=121). Disease-specific survival was found to be significantly shorter in patients with IGF2BP3 expressing clear cell carcinomas $(P=0.001$, Figure 3a). There was no significant difference in disease-specific survival between patients whose tumors did and those whose tumors did not express IGF2BP3 for high-grade serous (Figure 3b) or endometrioid subtypes (Figure 3c). In clear cell carcinoma only stage was a significant clinical risk factor in univariate analysis (data not shown) and therefore was introduced into a multivariable Cox proportional hazards regression model, together with IGF2BP3 status. For IGF2BP3 expression, a risk ratio of 2.9 (95\% confidence interval 1.45.8, Table 2) independent from stage was calculated.

To validate this finding, an independent cohort of 150 ovarian clear cell carcinomas from three other centers $(N=69$ from Johns Hopkins University, Baltimore, MD, USA, $N=42$ from University of Alberta, Edmonton, Canada, and $N=39$ from Memorial Sloan Cancer Center, New York, NY, USA) was assessed for IGF2BP3 expression. The IGF2BP3 expression rate was similar to the BC cohort, with 54.0 and $51.6 \%$ of clear cell carcinomas showing IGF2BP3 expression, respectively. Univariate and multivariate analyses confirmed the independent prognostic significance of IGF2BP3 expression for ovarian clear cell carcinoma in this series (Figure 3d and Table 2).

On the basis of these findings, we combined both series and calculated the risk ratio for ovarian clear cell carcinomas in stage I or II based on IGF2BP3 expression. Patients with IGF2BP3-expressing tumors exhibited a risk ratio of 2.8 (95\% confidence interval, 1.6-5.1) for disease-specific survival. The 5 -year disease-specific survival rate for women with IGF2BP3-negative ovarian clear cell carcinoma in stage I or II was $88 \%$ (standard error $3.5 \%$ ) and for IGF2BP3-positive tumors was $67 \%$ (standard error $4.9 \%)$.

\section{Discussion}

As there are no validated prognostic biomarkers for ovarian clear cell carcinoma, stage at diagnosis is the only information that clinicians can use to prognosticate at present. In this study we validated IGF2BP3 as a biomarker of prognostic significance for ovarian carcinomas of clear cell subtype, independent of stage.

Although IGF2BP3 is expressed in a variety of malignant neoplasms, including pulmonary small cell, ${ }^{19}$ endometrial, ${ }^{20}$ and cervical carcinomas, ${ }^{21}$ prognostic value has been shown only in renal clear cell carcinomas, ${ }^{8}$ other types of renal carcinomas, ${ }^{22}$ and low-stage urothelial carcinomas of the bladder. ${ }^{23}$ Validation of the association with prognosis in an independent case series has been exclusively 

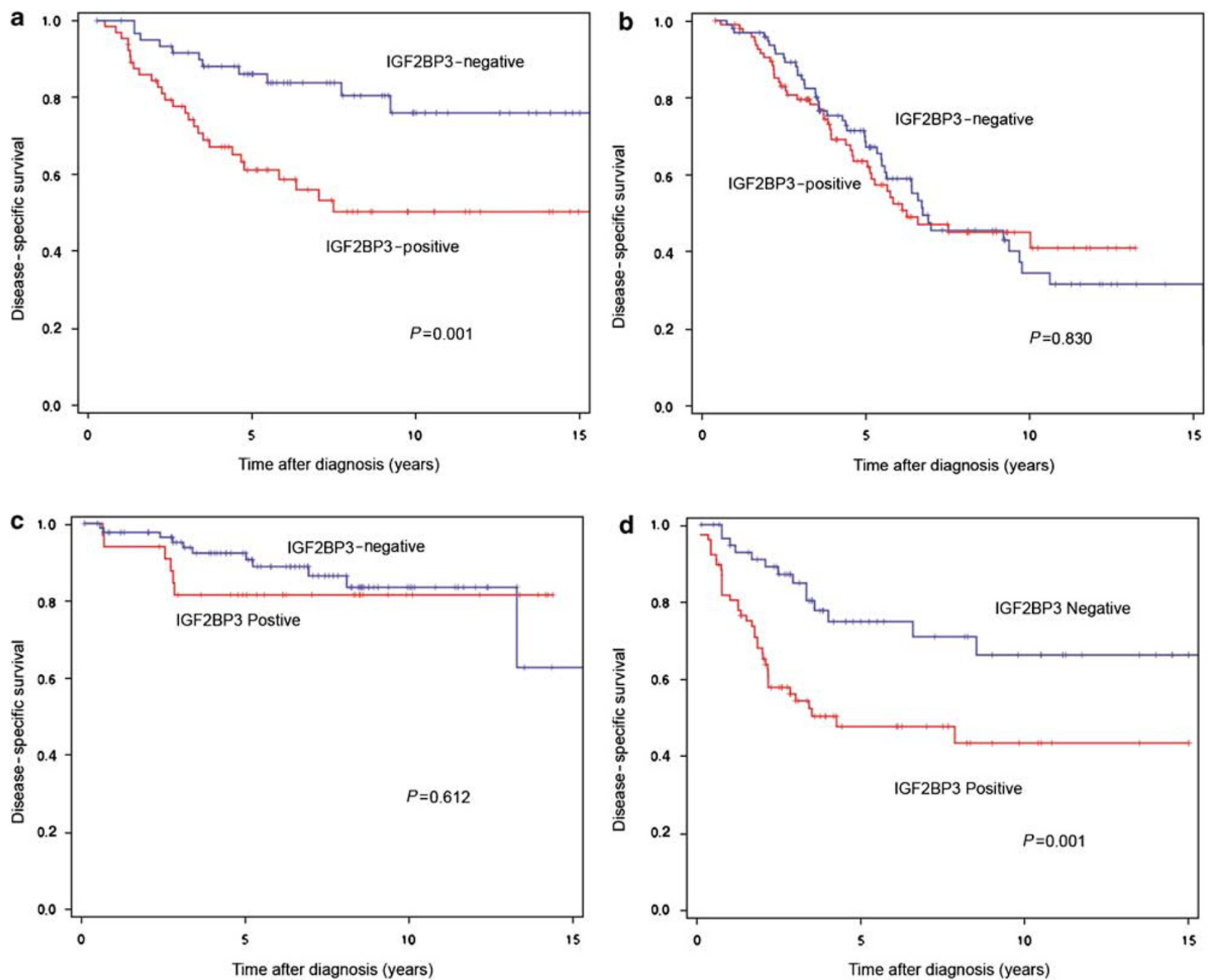

Figure 3 Kaplan-Meier analysis of disease-specific survival in ovarian carcinomas: (a) ovarian clear carcinomas from British Columbia $(N=128)$, (b) ovarian high-grade serous carcinomas $(N=198)$, (c) ovarian endometrioid carcinomas $(N=121)$, and $(\mathbf{d})$ ovarian clear cell carcinomas from the validation set $(N=150)$. $P$-values were calculated using the log-rank test.

Table 2 Multivariable analysis for disease-specific survival of the ovarian clear cell carcinomas

\begin{tabular}{|c|c|c|c|c|}
\hline \multirow[t]{2}{*}{ Variable } & \multicolumn{2}{|c|}{ British Columbia cohort } & \multicolumn{2}{|c|}{ Validation set } \\
\hline & $R R(95 \% C I)$ & $\mathrm{P}$-value & $R R(95 \% C I)$ & $\mathrm{P}$-value \\
\hline Stage (III/IV vs I/II) & $3.7(1.5-8.8)$ & 0.004 & $7.4(4.0-13.6)$ & $<0.001$ \\
\hline IGF2BP3 Expression $(+v s-)$ & $2.9(1.4-5.8)$ & 0.003 & $2.6(1.3-5.0)$ & 0.006 \\
\hline
\end{tabular}

CI, confidence interval; RR, risk ratio.

reported for renal clear cell carcinoma. ${ }^{9}$ The same prognostic significance is shown and validated here for ovarian clear cell carcinomas, but not other subtypes of ovarian carcinoma, suggesting a unique role of IGF2BP3 in these morphologically similar tumors. In contrast to renal clear cell carcinomas, in which abnormalities of the VHL/HIF1- $\alpha$ pathway have been identified in a majority of cases, ${ }^{24}$ the oncogenesis of ovarian clear cell carcinoma remains an enigma. ${ }^{25}$ IGF2BP3 has been postulated to increase expression levels of several oncogenes (IGF2, MYC) by stabilizing their mRNA. ${ }^{3}$

An attractive feature of IGF2BP3 as a biomarker is that its expression is found only in tumor tissue and is absent in normal adult tissue. This on/off phenomenon of expression makes staining interpretation straightforward in practice. The only normal tissue in which we observed IGF2BP3 expression is the placental intermediate trophoblast, which is the most invasive cell type during placental 
implantation; IGF2BP3 has been functionally implicated in cell migration. ${ }^{26}$ The lack of IGF2BP3 expression, which is normally expressed during embryogenesis, ${ }^{5}$ in normal adult tissue suggests that IGF2BP3 is epigenetically silenced in adult tissues. In ovarian carcinomas, there might be re-expression as a result of promoter hypomethylation, a common feature of ovarian carcinomas. ${ }^{27}$ The IGF2BP3 gene is located on chromosome $7 \mathrm{p}$ (at location $23316354-23476520$ ), a region not subject to frequent perturbation in ovarian carcinomas, ${ }^{28}$ making it unlikely that gene amplification is responsible for the observed IGF2BP3 expression in ovarian carcinoma. Therefore, we are currently testing the hypothesis that the IGF2BP3 promoter is hypomethylated in some ovarian clear cell carcinoma, and that this correlates with its expression levels. If so, IGF2BP3 could be regarded as a target for remethylating enzymes.

This is a retrospective study and a great majority of patients $(90 \%)$ received adjuvant therapy; hence, prognostic associations, strictly defined as tumor behavior after primary surgery, uninfluenced by different regimens of adjuvant therapy, cannot be assessed. Patients with ovarian clear cell carcinomas in this study were heterogeneously treated, with the large majority receiving chemotherapy with or without radiotherapy (eight different regimens that were given to at least five patients, and rare therapeutic regimens that were given to a few single patients, Supplementary Table 1). IGF2BP3 expression, however, showed the same trend for unfavorable prognosis in each treatment subgroup (data not shown). As only $10 \%$ of patients with ovarian clear cell carcinomas in this study were not treated with adjuvant therapy and this group consisted of patients in whom adjuvant therapy was either not advised or refused by the patient, we are not able retrospectively to compare adjuvant-treated patients with those receiving no adjuvant treatment.

A strength of this study is the large sample size (combined $N=278$ ) of ovarian clear cell carcinoma. The validation in an independent case series indicates that the prognostic value of IGF2BP3 stands up against potential patient selection biases, including different diagnostic and treatment standards. As stage III vs I/II is the strongest prognostic indicator in ovarian clear cell carcinoma, we examined the prognostic value of IGF2BP3 in stage I and II. Here we calculated a risk ratio of 2.8 for IGF2BP3-positive vs -negative ovarian clear cell carcinoma for disease-specific death combining both cohorts. IGF2BP3 expression might be used to stratify patients to adjuvant therapy. There is a subgroup of patients with clear cell carcinomas, namely stage I/II and IGF2BP3-negative, which has a good prognosis (5-year disease-specific survival rate of $88 \%$ ). Assuming the reported chemotherapy response rate for clear cell carcinomas (15-32\%) $)^{12,29}$ as an actual cure rate of one-third of the patients (which is undoubtedly an overestimate), we can estimate that from 100 patients 12 died despite treatment with adjuvant therapy and six patients were cured by chemotherapy; hence, 82 patients were treated without benefit. Considering the rate of severe side effects of chemotherapy, for example long-lasting grade III/IV peripheral sensory neurotoxicity reported in $7 \%$ of patients treated with carboplatin/paclitaxel, ${ }^{30}$ we would argue that assessment of IGF2BP3 expression in stage I or II ovarian clear cell carcinoma identifies patients in whom the risk/benefit ratio of current adjuvant therapy is so poor that consideration should be given to recommend against adjuvant therapy for some patients, a finding that now needs prospective validation.

\section{Acknowledgements}

This work was funded by the National Cancer Institute of Canada (\# 017051), the Michael Smith Foundation for Health Research Unit Grant (\#INRUA006045) to CBG, and by an unrestricted educational grant from Sanofi Aventis Canada. This work was supported by the Cheryl Brown Ovarian Cancer Outcomes Unit of the British Columbia Cancer Agency. MK received fellowship support from Eli Lilly Canada.

\section{Conflict of interest}

Betsy O Spaulding is employed by DAKO North America, which produced the antibody used in this study. She did not influence the study design, interpretation of data and the decision to submit the report for publication. The other authors have no conflicting financial interests to declare.

\section{References}

1 Nielsen FC, Nielsen J, Christiansen J. A family of IGF-II mRNA binding proteins (IMP) involved in RNA trafficking. Scand J Clin Lab Invest Suppl 2001; 234:93-99.

2 Liao B, Hu Y, Herrick DJ, et al. The RNA-binding protein IMP-3 is a translational activator of insulin-like growth factor II leader-3 mRNA during proliferation of human K562 leukemia cells. J Biol Chem 2005;280: 18517-18524.

3 Ioannidis P, Mahaira LG, Perez SA, et al. CRD-BP/IMP1 expression characterizes cord blood CD34+ stem cells and affects c-myc and IGF-II expression in MCF-7 cancer cells. J Biol Chem 2005;280:20086-20093.

4 Huttelmaier S, Zenklusen D, Lederer M, et al. Spatial regulation of beta-actin translation by Src-dependent phosphorylation of ZBP1. Nature 2005;438:512-515.

5 Mueller-Pillasch F, Pohl B, Wilda M, et al. Expression of the highly conserved RNA binding protein KOC in embryogenesis. Mech Dev 1999;88:95-99.

6 Kobel M, Weidensdorfer D, Reinke C, et al. Expression of the RNA-binding protein IMP1 correlates with poor 
prognosis in ovarian carcinoma. Oncogene 2007;26: 7584-7589.

7 Mueller-Pillasch F, Lacher U, Wallrapp C, et al. Cloning of a gene highly overexpressed in cancer coding for a novel KH-domain containing protein. Oncogene 1997;14:2729-2733.

8 Jiang Z, Chu PG, Woda BA, et al. Analysis of RNAbinding protein IMP3 to predict metastasis and prognosis of renal-cell carcinoma: a retrospective study. Lancet Oncol 2006;7:556-564.

9 Hoffmann NE, Sheinin Y, Lohse CM, et al. External validation of IMP3 expression as an independent prognostic marker for metastatic progression and death for patients with clear cell renal cell carcinoma. Cancer 2008;112:1471-1479.

10 Leitao Jr MM, Boyd J, Hummer A, et al. Clinicopathologic analysis of early-stage sporadic ovarian carcinoma. Am J Surg Pathol 2004;28:147-159.

11 Gilks CB, Ionescu D, Kalloger SE, et al. Tumor cell type can reproducibly diagnosed and is of independent prognostic significance in patients with maximally debulked ovarian carcinoma. Hum Pathol 2008;39:1239-1251.

12 Takano M, Kikuchi Y, Yaegashi N, et al. Clear cell carcinoma of the ovary: a retrospective multicentre experience of 254 patients with complete surgical staging. Br J Cancer 2006;94:1369-1374.

13 Tavasolli FA, Devilee P. World Health Organization Classification of Tumours.Tumours of the Breast and the Female Genital Organs. IARC Press-WHO, Lyon, France, 2003.

14 Köbel M, Kalloger SE, Carrick J, et al. A limited panel of immunomarkers can reliably distinguish between clear cell and high-grade serous carcinoma of the ovary. Am J Surg Pathol 2008, 30 September 2008; e-pub ahead of print.

15 Malpica A, Deavers MT, Lu K, et al. Grading ovarian serous carcinoma using a two-tier system. Am J Surg Pathol 2004;28:496-504.

16 Press JZ, De Luca A, Boyd N, et al. Ovarian carcinomas with genetic and epigenetic BRCA1 loss have distinct molecular abnormalities. BMC Cancer 2008;8:17 17.

17 Ishioka S, Sagae S, Terasawa K, et al. Comparison of the usefulness between a new universal grading system for epithelial ovarian cancer and the FIGO grading system. Gynecol Oncol 2003;89: 447-452.
18 Swenerton KD. Prognostic indices in ovarian cancer. Their significance in treatment planning. Acta Obstet Gynecol Scand Suppl 1992;155:67-74.

$19 \mathrm{Xu} \mathrm{H}$, Bourne PA, Spaulding BO, et al. High-grade neuroendocrine carcinomas of the lung express $\mathrm{K}$ homology domain containing protein overexpressed in cancer but carcinoid tumors do not. Hum Pathol 2007;38:555-563.

20 Zheng W, Yi X, Fadare O, et al. The oncofetal protein IMP3: a novel biomarker for endometrial serous carcinoma. Am J Surg Pathol 2008;32:304-315.

$21 \mathrm{Li} \mathrm{C}$, Rock KL, Woda BA, et al. IMP3 is a novel biomarker for adenocarcinoma in situ of the uterine cervix: an immunohistochemical study in comparison with p16(INK4a) expression. Mod Pathol 2007;20:242-247.

22 Jiang Z, Lohse CM, Chu PG, et al. Oncofetal protein IMP3: a novel molecular marker that predicts metastasis of papillary and chromophobe renal cell carcinomas. Cancer 2008;112:2676-2682.

23 Sitnikova L, Mendese G, Liu Q, et al. IMP3 predicts aggressive superficial urothelial carcinoma of the bladder. Clin Cancer Res 2008;14:1701-1706.

24 Costa LJ, Drabkin HA. Renal cell carcinoma: new developments in molecular biology and potential for targeted therapies. Oncologist 2007;12:1404-1415.

25 Tan DS, Kaye S. Ovarian clear cell adenocarcinoma: a continuing enigma. J Clin Pathol 2007;60:355-360.

26 Vikesaa J, Hansen TV, Jonson L, et al. RNA-binding IMPs promote cell adhesion and invadopodia formation. EMBO J 2006;25:1456-1468.

27 Barton CA, Hacker NF, Clark SJ, et al. DNA methylation changes in ovarian cancer: implications for early diagnosis, prognosis and treatment. Gynecol Oncol 2008;109:129-139.

28 Gorringe KL, Jacobs S, Thompson ER, et al. Highresolution single nucleotide polymorphism array analysis of epithelial ovarian cancer reveals numerous microdeletions and amplifications. Clin Cancer Res 2007;13:4731-4739.

29 Sugiyama T, Kamura T, Kigawa J, et al. Clinical characteristics of clear cell carcinoma of the ovary: a distinct histologic type with poor prognosis and resistance to platinum-based chemotherapy. Cancer 2000;88:2584-2589.

30 duBois A, Luck HJ, Meier W, et al. A randomized clinical trial of cisplatin/paclitaxel vs carboplatin/ paclitaxel as first-line treatment of ovarian cancer. J Natl Cancer Inst 2003;95:1320-1329.

Supplementary Information accompanies the paper on Modern Pathology website (http://www.nature.com/ modpathol) 